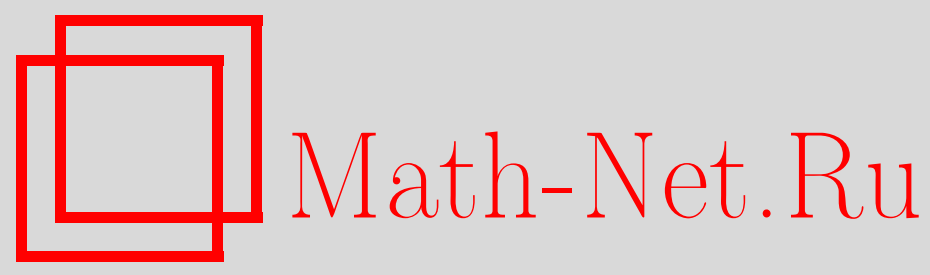

М. А. Овчинников, Представление гомеотопий тора простыми полиэдрами с краем, Матем. заметки, 1999, том 66, выпуск 4, 533-539

DOI: https://doi.org/10.4213/mzm1197

Использование Общероссийского математического портала Math-Net.Ru подразумевает, что вы прочитали и согласны с пользовательским соглашением http://www . mathnet.ru/rus/agreement

Параметры загрузки:

IP : 52.6 .47 .48

26 апреля 2023 г., 14:42:15

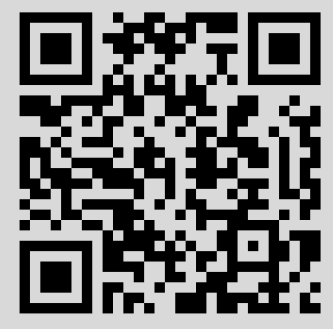




\title{
ПРЕДСТАВЛЕНИЕ ГОМЕОТОПИЙ ТОРА ПРОСТЫМИ ПОЛИЭДРАМИ С КРАЕМ
}

\author{
М.А. Овчинников
}

В 1991 году Тураев и Виро построили квантово-топологическое линейное представление групп классов отображений замкнутых поверхностей, сопоставляя отображениям поверхности в себя простые полиэдры с краем, состоящим из двух простых графов, разрезаюших поверхность на клетки. Выбор подходящего множества простых полиэдров сильно влияет на сложность вычислений представлений Тураева-Виро. В работе строятся такие простые полиэдры для случая тора, удобные тем, что все они получаются склейкой по краю из копий всего одного некоторого простого полиэдра.

Библиография: 14 названий.

1. Введение и формулировка результата. Впервые простые полиэдры были явно определены (под названием стандартных) в работе Каслера [1], которьй исследовал условия, при которых 3-многообразие восстанавливается по своему простому спайну. Свойства простых спайнов детально изучались С. В. Матвеевым [2], [3]. Эти результаты были существенно использованы Тураевьм и Виро при построении топологической квантовой теории поля на основе квантовых $6 j$-символов [4]. Представление отображений тора в себя простьпи полиэдрами, построенное в данной работе, непосредственно применимо для вычисления представления Тураева-Вирогрупшы классов отображения тора.

Кроме того, простые спайны как способ задания 3-многообразий используются в исследованиях геометрических структур на 3-многообразиях [5], [6], в исследованиях по знаменитым гипотезам Пуанкаре, Зимана, Эндрюса-Кертиса [7]-[9], в теории распознавания многообразий [10]. Последнее направление стимулируется бурным прогрессом персональной вычислительной техники. Простые спайны и, особенно, операции над ними вьгодно отличаются от других способов задания 3-многообразий экономностью и простотой компютерной реализации [11], [12].

Во всех исследованиях, где используется представление 3-многообразий простьми спайнами, могут найти применение методы построения простого спайна по другому представлению 3-многообразия: оснащенному зацеплению, диаграмме Хегора, разветвленному накрытию и т.п. K этому типу задач относится следующая задача, решаемая в этой работе. Пусть к данному 3-многообразию применена переклейка вдоль тора. Как найти простой спайн для полученного многобразия, если известны простой спайн исходного многообразия и гомеоморфизм переклейки? Частным случаем такого способа перестройки 3-многообразий является перестройка Дена (хирургия Дена), а именно, когда тор ограничивает полноторие.

Работа выполнена при поддержке Российского фонда фундаментальных исследований, грант № 99-01-00813. 
Результаты данной работы доставляют решение этой задачи для случая, когда пересечение исходного спайна с тором является тэта-кривой.

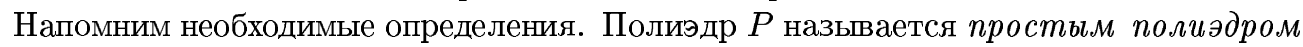
( краем), если у каждой его сингулярной точки линк является графом вида:

1) окружность с диаметром, либо

2) одномерный остов тетраэдра, либо

3) отрезок, либо

4) триод.

Множество точек типов 3) и 4) назьвается краем $P$ и обозначается через $\partial P$.

2 -полиэдр $P$ назьвается спайном связного компактного 3 -многообразия $M$, если для некоторого вложения $i: P \rightarrow M$ дополнение к $i(P)$ в $M$ гомеоморфно либо $\partial M \times[0,1)$, если край у $M$ непуст, либо открытому 3 -шару, если $M$ замкнуто. Если при этом полиэдр $Р$ простой, то он называется простым спайном.

Тәта-кривой называется граф, гомеоморфный окружности с диаметром. Тэта-кривую $\theta$ называем размеченной, если ее вершины и ребра пронумерованы. Для удобства в тексте вершины и ребра будем считать помеченньми вместо номеров символами $v_{1}, v_{2}$ и $e_{1}, e_{2}, e_{3}$ соответственно. Ребра считаем ориентированными от $v_{1} \mathrm{k} v_{2}$. Всюду далее, говоря о тэта-кривой в торе, будем подразумевать, что дополнение к тэта-кривой является открытым диском.

Полиэдр $P$ назьваем размеченным, если его край состоит из тэта-кривых и тэтакривые снабжены разметками (символически обозначаемыми $m_{1}$ и $m_{2}$ ). Если край полиэдра $P$ состоит из двух тэта-кривьх и тэта-кривые снабжены номерами - первая и вторая (помечены символами $\left.\theta_{1}, \theta_{2}\right)$, - то полиэдр $P$ назьваем ориентированным.

В данной работе рассматриваются только полиэдры с двумя тэта-кривыми на краю, поэтому для краткости под размеченностью будем подразумевать одновременно и ориентированность. Размеченньй полиэдр $P$ назьваем произведением размеченных полиэдров $P_{1}, P_{2}$, если его можно получить склеиванием копий полиэдров $P_{1}$ и $P_{2}$ путем отождествления второй тэта-кривой из $\partial P_{1}$ с первой из $\partial P_{2}$ в соответствии с разметкой. Данная работа посвящена изучению полиэдров, получающихся из следующего элементарного простого полиэдра.

ОПРЕДЕЛЕниЕ. Пусть $\theta_{1}$ и $\theta_{2}-$ две размеченные тэта-кривые, $a \vee b-$ букет ориентированных окружностей $a$ и $b$ с вершиной $*$ и клеточное отображение $f: \theta_{1} \sqcup \theta_{2} \rightarrow a \vee b$ действует по правилу

$$
\left.f\right|_{\theta_{1}}: e_{1} \rightarrow a, e_{2} \rightarrow b, e_{3} \rightarrow *,\left.\quad f\right|_{\theta_{2}}: e_{1} \rightarrow a, e_{2} \rightarrow-b, e_{3} \rightarrow * .
$$

Цилиндр $\operatorname{Cyl}(f)$ отображения $f$, рассматриваемьй вместе с разметкой тэта-кривых, назьваем полиәдром $J$.

ЗАмЕЧАНИЕ. Понятие разметки в этом определении следует трактовать в исходном смысле- без ориентированности. Но даже если размеченньй полиэдр $J$ понимается как ориентированньй, проблемы не возникает, благодаря его симметрии: все равно, какую из двух тэта-кривых объявить первой, а какую - второй.

Обозначим через $\langle J\rangle$ моноид, порожденньй полиэдром $J$ и размеченньпи полиэдрами, гомеоморфными $\theta \times I$. Единицей в нем является прямое произведение размеченной тэта-кривой на отрезок. Нетрудно убедиться, что полиэдр $J$ простой и, значит, все по-

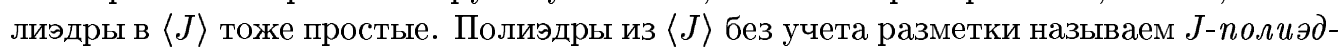
рами. В частности, $J$-полиэдром является $\theta \times I$. 
Грyпnой $\operatorname{Homeot}(F)$ гомеотопий поверхности $F$ называется факторгруппа группы гомеоморфизмов поверхности $F$ по подгруппе гомеоморфизмов, изотопных тождеству. Известно, что гомеотопии тора взаимно однозначно соответствуют автоморфизмам группы гомологий тора $[13$, теорема 5.15.5]. Это соответствие упрощает описание полиэдрального представления гомеотопий.

Пусть даны размеченный полиэдр $R \in\langle J\rangle$ и неразбивающее вложение $i: \theta \rightarrow T$ тэта-кривой, снабженной разметкой $m$, в тор $T$. Для изоморфизмов групп гомологий, индуцируемых этими полиэдром, вложением и разметками тэта-кривых, будем использовать следующие обозначения:

$$
\begin{gathered}
R_{*}: H_{1}\left(\theta_{1}\right) \rightarrow H_{1}\left(\theta_{2}\right), \quad i_{*}: H_{1}(\theta) \rightarrow H_{1}(T), \\
\left(m, m_{1}\right)_{*}: H_{1}(\theta) \rightarrow H_{1}\left(\theta_{1}\right), \quad\left(m, m_{2}\right)_{*}: H_{1}(\theta) \rightarrow H_{1}\left(\theta_{2}\right) .
\end{gathered}
$$

Определим соответствие $(i, m)_{*}:\langle J\rangle \rightarrow \operatorname{Homeot}\left(T^{2}\right)$ как сопоставляющее полиэдру $R$ гомеотопию $f$, если они определяют один и тот же автоморфизм гомологий тэта-кривой, т.е. $f$ обеспечивает коммутативность диаграммы изоморфизмов

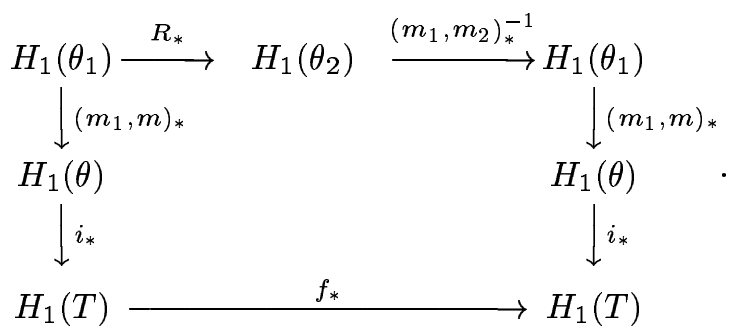

Оказывается, это соответствие является не только корректно определенным отображением, но и небольшой модификацией моноида превращается в биекцию. Обозначим через $\left\langle J \mid J^{2}\right\rangle$ фактор моноида $\langle J\rangle$ по отношению эквивалентности, порожденному соотношением $J^{2}=1$.

TЕОремА 1. Моноид $\left\langle J \mid J^{2}\right\rangle$ является группой, изоморфной группе $\operatorname{Homeot}\left(T^{2}\right)$.

Формально-алгебраические свойства $J$-полиэдров тесно связаны с их полезньми геометрическими свойствами, описьваемьми следующей теоремой.

ТЕОРема 2. Пусть 3-многообразия $M_{1}$ и $M_{2}$ получены из 3-многообразия $M_{0}$, возмохсно несвязного, склеиваниями $f_{1}, f_{2}: T_{1} \rightarrow T_{2}$ торов края д $M_{0}$. Предполохим, что полиәдр $P_{0} \subset M_{0}$, лежащие в нем тәта-кривые $\theta_{1}^{\prime}$ и $\theta_{2}^{\prime}$ являются неразбивающими тәта-кривыми в торах $T_{1}$ и $T_{2}$ соответственно и гомеоморфизмом $f_{1}$ отображаются друг на друга, а полиэдр $P_{1}$, полученный из полиэдра $P_{0}$ склейкой тәта-кривых $\theta_{1}^{\prime}, \theta_{2}^{\prime}$ посредством этого отоэсдествления, является спайном многообразия $M_{1}$. Тогда существуют $J$-полиэдр $R$ и гомеоморфизм приклейки $g_{2}: \partial R \rightarrow \theta_{1}^{\prime} \sqcup \theta_{2}^{\prime} \subset P_{0}$ такие, что полиэдр $P_{2}=R \cup_{g_{2}} P_{0}$ является спайном многообразия $M_{2}$.

ЗАмЕЧАНИЕ. Выбор разметки тЭта-кривой равносилен выбору гомеоморфизма с точностью до изотопии с некоторой избранной тэта-кривой $\Theta$. Размеченньй полиэдр является кобордизмом для тэта-кривой $\Theta$. Умножение размеченных полиэдров совершенно аналогично композиции кобордизмов. 
В [4] рассматривается категория $\underline{S}$, объекты которой - все 3 -валентные графы, а кобордизмы-все простыеполиэдры с краем. Соотношение $J^{2}=1$ естественно в том смысле, что простой спайн, преобразованньй посредством этого соотношения, остается простым спайном того же многообразия. В [4] рассматриваются преобразования, позволяюшие преобразовать друг в друга любые два простых спайна данного 3-многообразия, и определяется фактор $\underline{Q}$ категории $\underline{S}$ по порожденному этими преобразованиями отношению эквивалентности между кобордизмами в $\underline{S}$. Поэтому $\langle J\rangle$ может рассматриваться как подкатегория в $\underline{S}$, а $\left\langle J \mid J^{2}\right\rangle$ - как подкатегория в $\underline{Q}$, с той оговоркой, что в определении $\underline{S}$ и $\underline{Q}$ участвуют гомеоморфизмы, а в определении $\langle J\rangle$ и $\left\langle J \mid J^{2}\right\rangle$ - гомеоморфизмы с точностью до изотопии.

Обозначение умножения полиэдров выбрано слева-направо, хотя для композиции кобордизмов общепринято функциональное справа-налево. Так часто делается для удобства, когда операция умножения реально применяется в вычислениях, например, для записи умножения в группе преобразований или умножения матриц. Предлагаемая в данной работе алгебраическая конструкция разработана именно для реальных “вычислений” простых полиэдров (опробовано и используется), см. такжегипотезу в конце статьи.

2. Доказательство теоремы 1. Из-за сингулярных точек типа 2) обратимыми в $\langle J\rangle$ являются только полиэдры, гомеоморфные полиэдру $\theta \times I$. Состоящую из этих полиэдров подгруппу в $\langle J\rangle$ обозначаем $\langle\theta \times I\rangle$.

ПРЕДЛОЖЕНИЕ 1. Группа $\langle\theta \times I\rangle$ изоморфна группе $S_{3} \times \mathbb{Z}_{2}$.

Канонический изоморфизм естественен. Если ребра $1,2,3$ графа $\theta_{1} \subset P \in\langle\theta \times I\rangle$ проецируются в ребра $i_{1}, i_{2}, i_{3} \in\{1,2,3\} \subset \theta_{2}$ либо $\{-1,-2,-3\} \subset \theta_{2}$, то данному полиэдру сопоставляется элемент $\left(\left(i_{1}, i_{2}, i_{3}\right),+1\right) \in S_{3} \times \mathbb{Z}_{2}$ либо $\left(\left(i_{1}, i_{2}, i_{3}\right),-1\right) \in S_{3} \times \mathbb{Z}_{2}$ соответственно. В дальнейшем эти грушы не различаем ввиду очевидности данного изоморфизма. При этом для краткости единицу групшы обозначаем символом 1 , инволюцию из $\mathbb{Z}_{2}$ как подгруппы $S_{3} \times \mathbb{Z}_{2}$ обозначаем через $(-1)$, инволюции из $S_{3}$ обозначаем $(12),(23),(32)$, оставшиеся элементы $S_{3}$ обозначаем $(123),(132)$. Символ $(-123)$ соответствует элементу $((123),(-1))$ и т.п. Для размеченного полиэдра $P$ обозначение $-P$ равносильно $(-1) P$.

СЛЕДСТВИЕ 1. Полиәдры $J,(-1),(12),(23)$ порождают моноид $\langle J\rangle$.

СледСТВИЕ 2. Моноид $\left\langle J \mid J^{2}\right\rangle$ является группой.

ПРЕДЛОЖЕНИЕ 2. В полугруппе $\langle J\rangle$ имеют место следующие соотношения:

1) $(-1) J(-1)=J$;

2) $(12) J(12)=-J$.

ДокАЗАТЕЛьСтво. 1) Действие (-1) эквивалентно обращению ориентации ребер соответствующей тэта-кривой. Одновременная смена ориентаций ребер у обеих тэта-кривых у $J$ обеспечивает ту же самую склейку двух размеченных $\theta \times[0,1]$, что и в определении $J$.

2) Левую часть равенства можно истолковать согласно определению $J$ как полиэдр, склеенньй из $\theta_{1} \times I$ и $\theta_{2} \times I$ таким образом, что $e_{1} \times\{1\}$ из $\theta_{1} \times[0,1]$ отождествляется $\mathrm{c}-e_{1} \times\{0\}$ в $\theta_{2} \times[0,1]$ и $e_{2} \times\{1\}$ из $\theta_{1} \times[0,1]$ отождествляется с $e_{2} \times\{0\}$ в $\theta_{2} \times[0,1]$. Такой же размеченньй полиэдр задается правой частью равенства. 
СлЕДСТВИЕ 3. Элемент (-1) лехит в иентре моноида $\langle J\rangle$.

Изоморфизм $m_{*}: H_{1}(\theta) \rightarrow \mathbb{Z}+\mathbb{Z}$, индуцированньй разметкой $m$ тэта-кривой $\theta$, определим, сопоставляя циклу $e_{1} e_{3}^{-1} \subset \theta$ вектор-строку $(10) \in \mathbb{Z}+\mathbb{Z}$ и циклу $e_{2} e_{3}^{-1} \subset \theta$ вектор-строку $(01) \in \mathbb{Z}+\mathbb{Z}$. Таким образом, размеченный полиэдр $P$ индуцирует эндоморфизм $m_{2 *} P_{*} m_{1 *}^{-1}: \mathbb{Z}+\mathbb{Z} \rightarrow \mathbb{Z}+\mathbb{Z}$ и, тем самьм, индуцирует матричньй образ $A_{P}$ размеченного полиэдра $P$ в кольце матриц $M(2, \mathbb{Z})$. Справедливо равенство $A_{P_{1} P_{2}}=A_{P_{1}} A_{P_{2}}$ для любых $P_{1}, P_{2} \in\langle J\rangle$. Матрицы - образы полиэдра $J$ и размеченных полиэдров, гомеоморфных $\theta \times I,-$ вычисляем непосредственно.

ПРЕДЛОЖЕНИЕ 3. Матричные образы полиәдра J и размеченных полиәдров, гомеоморфных $\theta \times I$, имеют вид

$$
\begin{aligned}
& A_{1}=\left(\begin{array}{ll}
1 & 0 \\
0 & 1
\end{array}\right), \quad A_{-1}=\left(\begin{array}{cc}
-1 & 0 \\
0 & -1
\end{array}\right) \\
& A_{(23)}=\left(\begin{array}{cc}
1 & 0 \\
-1 & -1
\end{array}\right), \quad A_{(13)}=\left(\begin{array}{cc}
-1 & -1 \\
0 & 1
\end{array}\right), \quad A_{J}=\left(\begin{array}{cc}
1 & 0 \\
0 & -1
\end{array}\right) \text {. }
\end{aligned}
$$

Все вычисленные матрищы невырожденные. Известно, что эти матрицы порождают $G L(2, \mathbb{Z})$.

СлЕдСТВИЕ 4. Каждый размеченный полиэдр $P \in\langle J\rangle$ определяет автоморфизм группы $\mathbb{Z}+\mathbb{Z}$, и полученный гомоморфизм $A:\langle J\rangle \rightarrow G L(2, \mathbb{Z})$ является эпиморфизмом.

Так как $A_{J}^{2}=E$, то отображение $A$ индуцирует корректно определенньй гомоморфизм $A^{*}:\left\langle J \mid J^{2}\right\rangle \rightarrow G L(2, \mathbb{Z})$. Построим отображение $Q: G L(2, \mathbb{Z}) \rightarrow\left\langle J \mid J^{2}\right\rangle$ в обратную сторону.

Известно, что $G L(2, \mathbb{Z})$ на образующих

$$
R_{1}=\left(\begin{array}{ll}
0 & 1 \\
1 & 0
\end{array}\right), \quad R_{2}=\left(\begin{array}{cc}
-1 & 0 \\
1 & 1
\end{array}\right), \quad R_{3}=\left(\begin{array}{cc}
-1 & 0 \\
0 & 1
\end{array}\right), \quad Z=\left(\begin{array}{cc}
-1 & 0 \\
0 & -1
\end{array}\right)
$$

имеет копредставление [14, с. 126]

$$
\left\langle R_{1}, R_{2}, R_{3}, Z \mid R_{1}^{2}=R_{2}^{2}=R_{3}^{2}=E,\left(R_{1} R_{2}\right)^{3}=\left(R_{1} R_{3}\right)^{2}=Z, Z^{2}=E\right\rangle .
$$

Замечая, что $R_{1}=A_{(12)}, R_{2}=-A_{(23)}, R_{3}=-A_{J}, Z=A_{-1}$, определим $Q$ на образующих:

$$
R_{1} \rightarrow(12), \quad R_{2} \rightarrow-(23), \quad R_{3} \rightarrow-J, \quad Z \rightarrow-1 .
$$

Из свойств групшы $S_{3} \times \mathbb{Z}_{2}$ и предложения 2 легко следует, что для образов образующих вьполняются соответствуюшие соотношения, поэтому $Q$ корректно определено и, значит, является эпиморфизмом. Следовательно, $A^{*}$ и $Q$ - взаимно обратные изоморфизмы между $\left\langle J \mid J^{2}\right\rangle$ и $G L(2, \mathbb{Z})$. Теорема доказана.

3. Доказательство теоремы 2. Пусть символы $\theta_{1}, \theta_{2}, a \vee b, f$ означают то же, что и в определении полиэдра $J$. Будем считать графы $\theta_{1}, \theta_{2}, a \vee b$ лежащими в торах $T_{1}, T_{2}, T$ соответственно. Распространим по непрерывности клеточное отображение $f$ из определения полиэдра $J$ до отображения $F: T_{1} \sqcup T_{2} \rightarrow T$, являющегося гомеоморфизмом на каждом открытом диске $T_{i}-\theta_{i}, i=1,2$. Пара $(\operatorname{Cyl}(F), \operatorname{Cyl}(f))$, полученная из цилиндров отображений $F$ и $f$, состоит из утолщенного тора $T^{2} \times I$, где $I=[0,1]$, и размеченного полиэдра $J$. Обозначим ее через $\left(T^{2} \times I, J\right)$. 
Подполиэдр $P$ 3-многообразия $M$ назьвается собственно вложенным, если Int $P \subset$ Int $M$ и $\partial P \subset \partial M$. Так что пара $\left(T^{2} \times I, J\right)$ определяет собственное вложение полиэдра $J$ в утолщенньй тор.

Очевидным образом умножение размеченных полиэдров можно распространить до умножения пар вида (утолщенньй тор, собственно вложенный размеченньй полиэдр). Тогда пара $\left(T^{2} \times I, J\right)$ и пары $\left(T^{2} \times I, \theta \times I\right)$ порождают моноид, изоморфньй $\langle J\rangle$, а с соотношением $\left(T^{2} \times I, J\right)^{2}=\left(T^{2} \times I, \theta \times I\right)$, где $\theta \times I-$ прямоепроизведение размеченной тэта-кривой на отрезок, порождают групу, изоморфную $\left\langle J \mid J^{2}\right\rangle$.

Предположим, что на краях утолщенного тора $T^{2} \times I$ лежат размеченные тэта-кривые $\theta_{1}^{\prime}$ и $\theta_{2}^{\prime}$. Такую тройку обозначим через $\left(T^{2} \times I, \theta_{1}^{\prime}, \theta_{2}^{\prime}\right)$. Аналогично определяется умножение троек; тройки все, очевидно, обратимы и образуют групу, изоморфную группе $\left\langle J \mid J^{2}\right\rangle$. Разумеется, пары и тройки рассматриваются с точностью до гомеоморфизма, сохраняющего разметки тэта-кривых. Так что элементы моноида пар, дающие один и тот же элемент группы пар, “стиранием” полиэдра дают одну и ту же тройку.

Этот факт связывает свойства пар, троек и размеченных полиэдров из $\langle J\rangle$. Нам он понадобится для доказательства теоремы 2 и может быть сформулирован следующим образом.

ПРЕДЛОЖЕНИЕ 4. ДЛя любой тройки $\left(T^{2} \times I, \theta_{1}^{\prime}, \theta_{2}^{\prime}\right)$ найдутся полиэдр $P \in\langle J\rangle$ и собственное вложение $i: P \rightarrow T^{2} \times I$ такие, что $\partial i(P)=\theta_{1}^{\prime} \sqcup \theta_{2}^{\prime}$.

Докажем теорему 2 . Обозначим через $M^{\prime}$ многообразие, гомеоморфное $M_{2}$ и полученное из многообразия $M_{0}$ по сути той же склейкой, но с вклеенным утолщенным тором:

$$
M^{\prime}=M_{0} \sqcup\left(T_{1} \times[0,1]\right) /\left(\operatorname{id}_{1}: T_{1} \times\{0\} \rightarrow T_{1}, f^{\prime}: T_{1} \times\{1\} \rightarrow T_{2}\right),
$$

где $\mathrm{id}_{1}$ индуцировано тождеством $T_{1} \rightarrow T_{1}, f^{\prime}$ индуцировано гомеоморфизмом $f_{2}$. Обозначим через $p^{\prime}: P_{0} \rightarrow M^{\prime}$ вложение, индуцированное гомеоморфизмом вложения $M_{0} \subset M^{\prime}$. Имеем тройку $\left(T_{1} \times[0,1], p^{\prime}\left(\theta_{1}^{\prime}\right), p^{\prime}\left(\theta_{2}^{\prime}\right)\right)$.

Согласно предложению 4 найдутся полиэдр $R \in\langle J\rangle$ и собственное вложение $r: R \rightarrow$ $T_{1} \times[0,1]$, совмещающее края полиэдров $r(R)$ и $p^{\prime}\left(P_{0}\right)$.

Частичное отображение $r^{-1} p^{\prime}: \theta_{1}^{\prime} \sqcup \theta_{2}^{\prime} \rightarrow \theta_{1} \sqcup \theta_{2}$ обозначим через $g_{2}$, а результат склейки полиэдров $P_{0} \cup_{g_{2}} R$ - через $P_{2}$.

Учитывая, что $P_{1}-$ спайн и что $T_{1} \times[0,1]-r(R)$ является произведением открытого диска на отрезок, получаем, что $P_{2}$ - спайн с очевидным вложением $p: P_{2} \rightarrow M^{\prime}$, определяемым формулами $\left.p\right|_{P_{1}}=p^{\prime},\left.p\right|_{R}=r$. Поскольку $M^{\prime}$ гомеоморфно $M_{2}$, то $P_{2}-$ спайн многообразия $M_{2}$. Доказательство теоремы 2 завершено.

Условия теоремы 2 , вообще говоря, позволяют тэта-кривым $\theta_{1}^{\prime}, \theta_{2}^{\prime}$ не лежать на крае полиэдра $P_{0}$, проходить по внутренностям клеток, через вершины (сингулярные точки типа 2) ) и т.п. Практический интерес представляет следующий вариант этого результата.

СлЕДСТВИЕ 5. Если в условиях теоремы 2 полиәдр $P_{0}$ простой с краем $\partial P_{0}=$ $\theta_{1}^{\prime} \sqcup \theta_{2}^{\prime}$, то полиэдры $P_{1}$ и $P_{2}$ являются простыми спайнами многообразий $M_{1} и M_{2}$.

ЗАмЕчАниЕ. Гомеоморфизм склейки торов задают обычно матрицей из $G L(2, \mathbb{Z})$. Изоморфизмы $G L(2, \mathbb{Z}) \cong\left\langle J \mid J^{2}\right\rangle \cong \operatorname{Homeot}\left(T^{2}\right)$, построенные в работе, дают конструктивньй метод подбора полиэдра $R \in\langle J\rangle$ по матрище гомеоморфизма. Из таких полиэдров легко выбрать содержащий минимально возможное число вершин - критерием минимальности (неминимальности) служит отсутствие (наличие) в полиэдре 2-угольников. Из анализа построенного копредставления для $\left\langle J \mid J^{2}\right\rangle$ несложно выводится, 
что для любого вложения пары тэта-кривых в края утолщенного тора минимальный $J$-полиэдр единственен.

ГИПОТЕЗА. Для любой пары тәта-кривых на краях утолщенного тора простой полиәдр, имеющий минимально возможнное число вершин среди полиэдров с краем, состоящим из данных тэта-кривых, и разрезающих утолщенный тор до шара, является $J$-полиәдром.

Компьютерньм перебором проверена (“доказана") эта общая минимальность $J$-полиэдров без 2-угольников с числом вершин до восьми включительно.

Автор благодарит С. В. Матвеева за постановку задач и внимание к работе.

\section{СПИСОК ЦИТИРОВАННОЙ ЛИТЕРАТУРЫ}

[1] Casler B. G. An embedding theorem for connected 3-manifolds with boundary // Proc. Amer. Math. Soc. 1965. V. 16. P. 559-566.

[2] Матвеев С. В. Специальные остовы кусочно линейных многообразий // Матем. сб. 1973. T. 92. № 2. C. $282-293$.

[3] Матвеев С. В. Универсальные 3-деформации специальных полиэдров // УМН. 1987. Т. 42. № 3. C. 193-194.

[4] Turaev V. G., Viro O. Y. State sum invariants of 3-manifolds and quantum $6 j$-symbols // Topology. 1992. V. 31. P. 865-902.

[5] Матвеев С. В., Фоменко А. Т. Изоэнергетические поверхности гамильтоновых систем, перечисление трехмерных многообразий в порядке возрастания их сложности и вычисление объемов замкнутых гиперболических многообразий // УМН. 1988. Т. 43. № 1. С. 5-22.

[6] Benedetti R., Petronio C. Branched standard spines of 3-manifolds. Lecture Notes in Math. V. 1653. New York: Springer, 1997.

[7] Матвеев С. В. Гипотеза Зимана для неутолщаемых специальных полиэдров эквивалентна гипотезе Эндрюса-Кертиса // Сиб. матем. ж. 1987. Т. 28. №6. С. 66-80.

[8] Матвеев С. В. Преобразования специальных спайнов и гипотеза Зимана // Изв. АН СССР. Сер. матем. 1987. T. 51. №5. C. 1104-1116.

[9] Gillman D., Rolfsen D. The Zeeman Conjecture for standard spines is equivalent to the Poincaré Conjecture // Topology. 1983. V. 22. №3. P. 315-323.

[10] Matveev S. V. Complexity theory of three-dimensional manifolds // Acta Appl. Math. 1990. V. 19. P. 101-130.

[11] Matveev S. V. On a computer recognition of 3-manifolds. MSRI Preprint No. 1997-028: MSRI, 1997.

[12] Adams C. SNAPPEA: The Week's hyperbolic 3-manifolds program // Notices Amer. Math. Soc. 1990. V. 37. P. 273-275.

[13] Цишанг Х., Фогт Э., Колдевай Х.-Д. Поверхности и разрьвные группы. М.: Наука, 1988.

[14] Коксетер Г. С. М., Мозер У. О. Порождающие элементы и определяющие соотношения. М.: Наука, 1980.

Челябинский государственный университет

Поступило

E-mail: ovch@csu.ac.ru

03.09 .98

Исправленный вариант

09.07.99 